\title{
Maternal plasma folate impacts differential DNA methylation in an epigenome-wide meta-analysis of newborns
}

Bonnie R. Joubert ${ }^{1, \star}$, Herman T. den Dekker ${ }^{2,3,4, \star}$, Janine F. Felix ${ }^{2,4,5}$, Jon Bohlin ${ }^{6}$, Symen Ligthart ${ }^{4}$, Emma Beckett 7,8 , Henning Tiemeier ${ }^{4,9}$, Joyce B. van Meurs ${ }^{10}$, Andre G. Uitterlinden ${ }^{4,10}$, Albert Hofman ${ }^{4}$, Siri E. Håberg ${ }^{6}$, Sarah E. Reese ${ }^{1}$, Marjolein J. Peters ${ }^{10}$, Bettina Kulle Andreassen ${ }^{11}$, Eric A.P. Steegers ${ }^{12}$, Roy M. Nilsen ${ }^{13}$, Stein E. Vollset 6,14 , Øivind Midttun ${ }^{15}$, Per M. Ueland ${ }^{16,17}$, Oscar H. Franco ${ }^{4}$, Abbas Dehghan ${ }^{4}$, Johan C. de Jongste ${ }^{3}$, Michael C. Wu ${ }^{18}$, Tianyuan Wang ${ }^{1}$, Shyamal D. Peddada ${ }^{1}$, Vincent W.V. Jaddoe ${ }^{2,4,5}$,

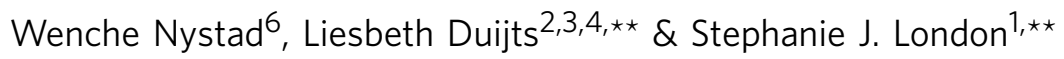

Folate is vital for fetal development. Periconceptional folic acid supplementation and food fortification are recommended to prevent neural tube defects. Mechanisms whereby periconceptional folate influences normal development and disease are poorly understood: epigenetics may be involved. We examine the association between maternal plasma folate during pregnancy and epigenome-wide DNA methylation using Illumina's HumanMethyl450 Beadchip in 1,988 newborns from two European cohorts. Here we report the combined covariate-adjusted results using meta-analysis and employ pathway and gene expression analyses. Four-hundred forty-three CpGs (320 genes) are significantly associated with maternal plasma folate levels during pregnancy (false discovery rate 5\%); 48 are significant after Bonferroni correction. Most genes are not known for folate biology, including APC2, GRM8, SLC16A12, OPCML, PRPH, LHX1, KLK4 and PRSS21. Some relate to birth defects other than neural tube defects, neurological functions or varied aspects of embryonic development. These findings may inform how maternal folate impacts the developing epigenome and health outcomes in offspring.

\footnotetext{
${ }^{1}$ Division of Intramural Research, National Institute of Environmental Health Sciences, National Institutes of Health, Department of Health and Human Services, Research Triangle Park, North Carolina 27709, USA. ${ }^{2}$ The Generation R Study Group, Erasmus MC, University Medical Center Rotterdam, Rotterdam 3000 CA, Netherlands. ${ }^{3}$ Department of Pediatrics, Division of Respiratory Medicine, Erasmus MC, University Medical Center Rotterdam, Rotterdam 3000 CA, Netherlands. ${ }^{4}$ Department of Epidemiology, Erasmus MC, University Medical Center Rotterdam, Rotterdam 3000 CA, Netherlands. ${ }^{5}$ Department of Pediatrics, Erasmus MC, University Medical Center Rotterdam, Rotterdam 3000 CA, Netherlands. ${ }^{6}$ Norwegian Institute of Public Health, Oslo 0403, Norway. ${ }^{7}$ Department of Applied Sciences, School of Environmental and Life Sciences, University of Newcastle, Ourimbah, New South Wales 2258, Australia. ${ }^{8}$ Food and Nutrition Flagship, CSIRO, North Ryde, New South Wales 2113, Australia. ${ }^{9}$ Department of Psychiatry, Erasmus MC, University Medical Center Rotterdam, Rotterdam 3000 CA, Netherlands. ${ }^{10}$ Department of Internal Medicine, Erasmus MC, University Medical Center Rotterdam, Rotterdam 3000 CA, Netherlands. ${ }^{11}$ Department of Clinical Molecular Biology, Institute of Clinical Medicine, University of Oslo, Oslo 0316, Norway. ${ }^{12}$ Department of Obstetrics and Gynaecology, Erasmus MC, University Medical Center Rotterdam, Rotterdam 3000 CA, Netherlands. ${ }^{13}$ Department of Research and Development, Centre for Clinical Research, Haukeland University Hospital, Bergen 5021, Norway. ${ }^{14}$ Department of Global Public Health and Primary Care, University of Bergen, Bergen 5018, Norway. ${ }^{15}$ Bevital A/S, Laboratoriebygget, Bergen 5018, Norway. ${ }^{16}$ Department of Clinical Science, University of Bergen, Bergen 5018, Norway. ${ }^{17}$ Laboratory of Clinical Biochemistry, Haukeland University Hospital, Bergen 5018, Norway. ${ }^{18}$ Public Health Sciences Program, Fred Hutchinson Cancer Research Center, Seattle, Washington 98109, USA. * These authors contributed equally to this work. ${ }^{\star \star}$ These authors jointly supervised this work. Correspondence and requests for materials should be addressed to B.R.J. (email: joubertbr@niehs.nih.gov).
} 
F olate (vitamin B9) is vital for fetal development. Folic acid supplementation at $0.4 \mathrm{mg}$ per day or higher is recommended worldwide before and in the very early stages of pregnancy to reduce the incidence of neural tube defects (NTDs). Over 50 countries have introduced programs to fortify the food supply with folic acid to increase folate levels in women of childbearing age ${ }^{1}$. Rates of NTDs have clearly decreased following fortification $^{2}$ and there is increasing interest in the possibility that higher maternal folate prevents additional birth defects including oral clefts, cardiac defects and others ${ }^{3}$. A large international trial has been launched of supplementation with $4 \mathrm{mg}$ versus the standard $0.4 \mathrm{mg}$ to attempt to address these questions ${ }^{3}$.

Other beneficial effects of higher maternal folate levels have been reported in humans. These include reduced risk of low birth weight, pre-term delivery, language delay, leukaemia, childhood brain tumours and autism, although the evidence is inconsistent ${ }^{4,5}$. In the United States, food fortification has led to an increase in folate intake twice as large as anticipated ${ }^{6}$, and therefore concern has been raised about possible adverse effects, such as cancer in adults, as a result of this population-wide intervention ${ }^{1}$. Furthermore, higher folic acid intake during pregnancy has been associated with an increased risk of childhood retinoblastoma and early respiratory illness 4 .

The mechanisms whereby folic acid prevents NTDs and potentially other birth defects and later health outcomes are poorly understood ${ }^{7}$ but could involve epigenetic changes. Folate is a critical component in the one-carbon metabolism pathway providing methyl groups for a range of biochemical reactions, including methylation of $\mathrm{DNA}^{8}$. DNA methylation is an important epigenetic determinant of gene expression, and differential methylation has been associated with multiple diseases ${ }^{9}$. Periconceptional maternal folate levels may alter methylation patterns established in utero that are vital for fetal development, which could impact later health outcomes in the offspring.

In mouse models, in utero dietary methyl donor supplementation has been associated with altered methylation patterns and disease phenotypes ${ }^{4}$. The brains of human fetuses with NTDs had lower global methylation compared with controls, which was positively correlated with maternal folate levels ${ }^{10}$. With respect to gene-specific differential methylation, perinatal folate has also been associated with differential methylation in specific imprinted genes, such as IGF2 and H19, in offspring, but reported results are inconsistent ${ }^{11}$. The only published study using a platform with reasonable genome-wide coverage, the Illlumina HumanMethyl 450 Beadchip ( $450 \mathrm{~K}$ ), investigated 23 subjects and reported that folic acid supplementation during pregnancy was related to differential methylation upstream of the gene ZFP57, which plays a central role in the regulation and maintenance of imprinting ${ }^{12}$.

Some countries, such as Norway and the Netherlands, do not fortify the food supply with folic acid. These populations may be particularly useful for examining the biological implications of periconceptional folic acid supplementation on offspring health, as greater variability in the dose and the source of folate may exist compared with fortified populations.

To better understand the biological implications of folate status on the developing fetus, we examine the association between maternal plasma folate during pregnancy and epigenome-wide differential DNA methylation in newborn cord blood using the Illumina HumanMethyl450 (450 K) Beadchip. We include 1,988 newborns from two European pregnancy cohorts of Caucasian ancestry, the Norwegian Mother and Child Cohort Study (MoBa), and the Generation R Study (Generation R). We combine results using meta-analysis. Secondary pathway analyses and gene expression analyses are also explored.

\section{Results}

Study characteristics. In MoBa participants $(N=1,275)$, maternal plasma folate levels ranged from 1.6 to $53.2 \mathrm{nmoll}^{-1}$ $($ mean $=11.9)$. The maternal plasma folate levels in Generation $\mathrm{R}$ $(N=713)$ ranged from 4.1 to $45.3 \mathrm{nmoll}^{-1} \quad$ (mean $=20.3$; Table 1). The mean maternal age was $\sim 30$ years for both cohorts. Approximately, $15 \%$ of MoBa mothers and $25 \%$ of Generation $\mathrm{R}$ mothers smoked during the pregnancy and over $60 \%$ obtained college or more advanced levels of education in both studies (Table 1).

Meta-analysis. Our meta-analysis of the association between maternal plasma folate levels during pregnancy and differential DNA methylation in newborn cord blood, adjusted for covariates, resulted in 443 false discovery rate (FDR)-significant CpGs (Benjamini and Hochberg FDR-corrected $P\left(P_{\mathrm{BH}}\right)<0.05$; Fig. 1). Genes with two or more FDR-significant $\mathrm{CpGs}$, where at least one $\mathrm{CpG}$ was within the gene, were prioritized for further discussion (Table 2). Results for all FDR-significant CpGs are shown in Supplementary Data 1 (sorted by the uncorrected $P$ value) and Supplementary Data 2 (sorted by chromosome and position). The vast majority of the FDR-significant CpGs were robust to covariate adjustment as well as adjustment for cell type; coefficients from the unadjusted, covariate-adjusted, and covariate- and cell-type-adjusted models were in the same direction and had a similar magnitude of effect (Supplementary Data 1 and 2). More detailed gene information is provided in Supplementary Table 1. The genomic inflation factor (lambda) ${ }^{13}$ values for the unadjusted, covariate-adjusted, and covariate- and cell-typeadjusted models were 0.96, 1.07 and 1.16, respectively (Supplementary Figs 1-3). Among the 443 FDR-significant CpGs in the covariate-adjusted meta-analysis model, increasing levels of maternal plasma folate during pregnancy were associated with decreased methylation of 416 (94\%) and increased methylation of 27 (6\%) CpGs. There were 48 CpGs that also met the strict Bonferroni threshold for statistical significance $\left(P<1.19 \times 10^{-7}\right.$, correcting for 419,905 tests $)$. The direction of effects for the statistically significant CpGs was largely consistent in the MoBa and Generation $\mathrm{R}$ populations (Table 2; Supplementary Data 1 and 2).

Sensitivity analyses. We considered whether vitamin B12, a co-factor with folate in one-carbon metabolism, contained in most multivitamins, along with other $\mathrm{B}$ vitamins such as B6 and riboflavin, might confound associations between folate and methylation. Vitamin B12 and folate levels were modestly positively correlated (Spearman correlation 0.11 in MoBa, 0.14 in Generation $\mathrm{R}, P<0.001$ for both). When we adjusted for vitamin $\mathrm{B} 12$, the coefficients for folate in relation to methylation changed only minimally (median change 4.9\%, 25-75th percentile $2.3-8.2 \%, N=1,933$ subjects). In addition to the consistency of effect estimates after adjustment, results remained statistically significant for $376(85 \%)$ at Bonferroni correction for 443 tests, $P<1.13 \times 10^{-4}$, and all 443 CpGs had $P<9 \times 10^{-4}$ (Supplementary Data 3). Thus, vitamin B12 does not confound the folate-methylation associations we observed.

Women with higher folate levels, which largely reflect supplement use, might be more likely to take multivitamins and/or separate supplements such as cod liver or fish oils that are common in Norway. However, vitamin D (total of D2 and D3) levels were modestly correlated with folate levels (Spearman correlation coefficient $=0.14$ in $\mathrm{MoBa}, 0.23$ in Generation $\mathrm{R}$, $P<0.001$ for both cohorts). Adjustment for vitamin $\mathrm{D}$ only minimally altered effect estimates for folate in relation to methylation (median absolute value of change $7.3 \%, 25-75$ th 
Table 1 | Descriptive characteristics of the MoBa and Generation R study populations.

\begin{tabular}{|c|c|c|c|}
\hline Variable & Category & MoBa N (\%) & Generation R N (\%) \\
\hline \multirow[t]{4}{*}{ Maternal plasma folate $\left(\mathrm{nmoll}^{-1}\right)$} & Min-max & $1.6-53.2$ & $4.1-45.3$ \\
\hline & Mean & 11.9 & 20.3 \\
\hline & Median & 9.1 & 19.6 \\
\hline & 25-75th percentile & $6.2-16.0$ & $13.3-26.4$ \\
\hline \multirow[t]{4}{*}{ Log-transformed maternal plasma folate } & Min-max & $0.48-4.0$ & $1.4-3.8$ \\
\hline & Mean & 2.3 & 2.9 \\
\hline & Median & 2.2 & 3.0 \\
\hline & 25-75th percentile & $1.8-2.8$ & $2.6-3.3$ \\
\hline Maternal age (years) & Mean (s.d.) & $29.9(4.3)$ & $31.5(4.1)$ \\
\hline \multirow[t]{5}{*}{ Maternal education level } & Less than secondary school & $96(7.5)$ & $14(1.8)$ \\
\hline & Secondary school completion & $415(32.3)$ & $267(34.3)$ \\
\hline & Some college or university & $566(44.1)$ & $203(26.0)$ \\
\hline & 4 Years or more of college/university & $206(16.1)$ & $296(37.9)$ \\
\hline & Missing & 6 & 10 \\
\hline \multirow[t]{5}{*}{ Parity $^{\star}$} & 0 & $537(41.7)$ & $479(60.7)$ \\
\hline & 1 & $511(39.6)$ & $240(30.4)$ \\
\hline & 2 & 179 (13.9) & $63(8.0)$ \\
\hline & $3+$ & $62(4.8)$ & $7(0.9)$ \\
\hline & Missing & 0 & 1 \\
\hline \multirow[t]{3}{*}{ Maternal smoking during pregnancy } & No & $1098(85.2)$ & $541(75.6)$ \\
\hline & Yes & $191(14.8)$ & $175(24.5)$ \\
\hline & Missing & 0 & 74 \\
\hline
\end{tabular}

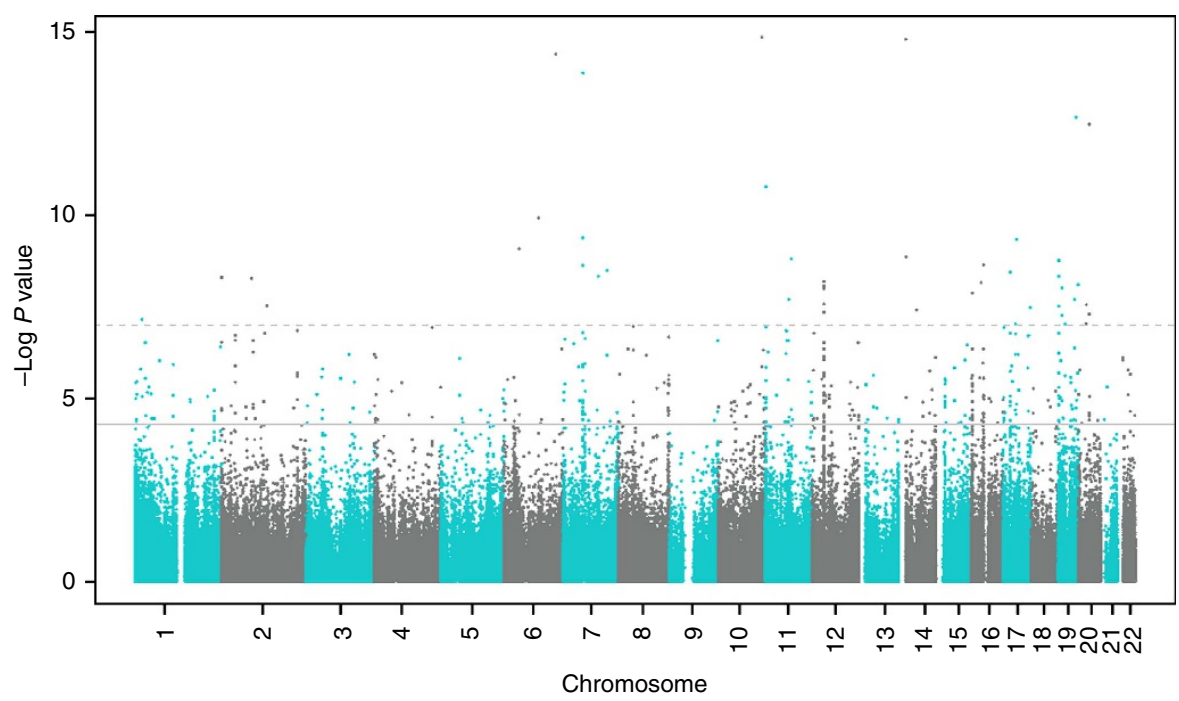

Figure 1 | Association between maternal plasma folate and DNA methylation in newborn cord blood: meta-analysis results for MoBa ( $N=1,275)$ and Generation $\mathbf{R}(\mathbf{N}=\mathbf{7 1 3})$ cohorts. The uncorrected -log10( $P$ values) are plotted by $C p G$ genomic position. Multiple testing was accounted for using the false discovery rate (FDR) procedure by Benjamini and Hochberg. A total of 443 CpGs were considered FDR significant (solid horizontal line); 48 CpGs were also Bonferroni significant (dashed horizontal line).

percentile 3.3-12.3\%). Despite the reduction in power due to the smaller sample size for these adjusted analyses $(N=1,664), 70 \%$ of CpGs significantly related to folate in the main model remained Bonferroni significant after adjustment for vitamin D (308 with $P<1.13 \times 10^{-4}$; Supplementary Data 3).

We performed additional analyses adjusting for two singlenucleotide polymorphisms (SNPs) in the MTHFR gene that influence one-carbon metabolism and are correlated with plasma folate: rs1801133 and rs1801131 (refs 14,15). These SNPs are in moderate linkage disequilibrium with each other $\left(r^{2}=0.20-21\right.$ in the two studies). Adjustment for these two SNPs made little difference in the effect estimates compared with the main model; median change in coefficient $=3.8 \%(25-75$ th percentile $=2.0$ $6.9 \%$ ) and $85 \%$ of CpGs remained statistically significant despite reduction in sample size to $1,880\left(P<1.13 \times 10^{-4}\right.$, correction for 443 tests). Thus, these genetic variants do not confound the relationship between folate and methylation.

Homocysteine, unlike folate or vitamin B12, is not a nutrient that plays a role as a methyl donor or carrier, but is a product formed during transmethylation in the one-carbon metabolism cycle. It could be regarded as an intermediate on the causal pathway between folate and methylation. In addition, like plasma 
Table 2 | Selected loci with differential methylation in cord blood in relation to maternal folate.

\begin{tabular}{|c|c|c|c|c|c|c|c|c|c|c|c|c|c|c|}
\hline \multirow[t]{2}{*}{ CHR } & \multirow[t]{2}{*}{ Position } & \multirow[t]{2}{*}{ CpG } & \multirow[t]{2}{*}{ Gene } & \multirow{2}{*}{$\begin{array}{l}\text { Gene } \\
\text { group }\end{array}$} & \multicolumn{3}{|c|}{ MoBa } & \multicolumn{3}{|c|}{ Generation R } & \multirow[b]{2}{*}{$\begin{array}{l}\text { Weighted } \\
\text { mean beta }\end{array}$} & \multicolumn{3}{|c|}{ Meta-analysis } \\
\hline & & & & & Coef & s.e. & $P$ & Coef & s.e. & $P$ & & Coef & s.e. & $P$ \\
\hline 7 & 126698829 & cg15908975 & GRM8 & Body & -0.012 & 0.003 & $8.26 E-06$ & -0.015 & 0.007 & $2.46 \mathrm{E}-02$ & 0.54 & -0.012 & 0.002 & $6.76 E-07$ \\
\hline 7 & 126889015 & cg18574254 & GRM8 & 5'-UTR & -0.010 & 0.002 & $3.25 E-06$ & -0.011 & 0.003 & $2.40 E-04$ & 0.84 & -0.011 & 0.002 & $3.27 E-09$ \\
\hline 10 & 91296252 & cg22591480 & SLC16A12 & TSS1500 & -0.009 & 0.002 & $1.96 \mathrm{E}-04$ & -0.008 & 0.003 & $2.33 \mathrm{E}-02$ & 0.85 & -0.008 & 0.002 & $1.34 \mathrm{E}-05$ \\
\hline 10 & 91296311 & cg14920044 & SLC16A12 & TSS1500 & -0.011 & 0.003 & $6.45 E-05$ & -0.011 & 0.005 & $2.31 E-02$ & 0.78 & -0.011 & 0.003 & $4.31 E-06$ \\
\hline 11 & 132951838 & cg24829292 & OPCML & Body & 0.008 & 0.003 & $9.45 E-03$ & 0.014 & 0.004 & $7.55 E-05$ & 0.44 & 0.010 & 0.002 & $6.60 E-06$ \\
\hline 11 & 132951861 & cg22629528 & OPCML & Body & 0.015 & 0.005 & $5.45 E-03$ & 0.031 & 0.009 & $4.54 E-04$ & 0.60 & 0.019 & 0.005 & $2.91 E-05$ \\
\hline 11 & 132951950 & cg26283170 & OPCML & Body & 0.006 & 0.002 & $9.46 \mathrm{E}-03$ & 0.015 & 0.004 & $4.58 E-05$ & 0.54 & 0.009 & 0.002 & $1.30 E-05$ \\
\hline 12 & 49689685 & cg24804179 & PRPH & Body & -0.007 & 0.002 & $3.73 E-05$ & -0.007 & 0.004 & $8.72 E-02$ & 0.20 & -0.007 & 0.002 & $8.05 E-06$ \\
\hline 12 & 49690254 & cg05775627 & $P R P H$ & Body & -0.006 & 0.002 & $3.61 \mathrm{E}-03$ & -0.007 & 0.002 & $7.87 E-04$ & 0.63 & -0.007 & 0.002 & $1.01 E-05$ \\
\hline 12 & 49692283 & cg16010628 & PRPH & 3'-UTR & -0.006 & 0.001 & $8.58 E-06$ & -0.002 & 0.003 & $4.51 E-01$ & 0.29 & -0.005 & 0.001 & $1.73 E-05$ \\
\hline 16 & 2866901 & cg05635274 & PRSS21 & TSS1500 & 0.008 & 0.002 & $1.79 E-04$ & 0.013 & 0.005 & $5.05 E-03$ & 0.82 & 0.009 & 0.002 & $4.77 E-06$ \\
\hline 16 & 2867051 & cg02296564 & PRSS21 & TSS200 & 0.011 & 0.003 & $7.43 E-04$ & 0.011 & 0.004 & $2.63 E-03$ & 0.64 & 0.011 & 0.003 & $6.21 E-06$ \\
\hline 16 & 2867434 & cg22730830 & PRSS21 & Body & 0.012 & 0.003 & $2.69 E-04$ & 0.016 & 0.005 & $4.02 E-03$ & 0.59 & 0.013 & 0.003 & $3.99 E-06$ \\
\hline 16 & 2867446 & cg01232511 & PRSS21 & Body & 0.012 & 0.004 & $9.50 E-04$ & 0.020 & 0.006 & $2.32 E-03$ & 0.67 & 0.014 & 0.003 & $1.23 E-05$ \\
\hline 17 & 35285205 & cg10612259 & LHX1 & & -0.009 & 0.003 & $1.91 \mathrm{E}-04$ & -0.015 & 0.004 & $5.54 \mathrm{E}-05$ & 0.47 & -0.011 & 0.002 & $9.10 E-08$ \\
\hline 17 & 35285295 & cg01965477 & LHX1 & & -0.002 & 0.001 & $5.26 E-04$ & -0.003 & 0.001 & $8.80 E-03$ & 0.06 & -0.002 & 0.001 & $2.09 E-05$ \\
\hline 19 & 1453909 & cg11775595 & $A P C 2$ & Body & -0.017 & 0.003 & $8.04 E-07$ & -0.010 & 0.005 & $3.57 E-02$ & 0.44 & -0.015 & 0.003 & $1.64 \mathrm{E}-07$ \\
\hline 19 & 1456246 & cg14907738 & $A P C 2$ & Body & -0.006 & 0.001 & $3.59 E-05$ & -0.005 & 0.003 & $9.34 \mathrm{E}-02$ & 0.22 & -0.006 & 0.001 & $8.57 E-06$ \\
\hline 19 & 1456337 & cg27150178 & $A P C 2$ & Body & -0.009 & 0.002 & $6.01 E-06$ & -0.007 & 0.003 & $3.00 E-02$ & 0.32 & -0.009 & 0.002 & $5.81 E-07$ \\
\hline 19 & 1456886 & cg03165176 & $A P C 2$ & Body & -0.012 & 0.003 & $1.96 \mathrm{E}-04$ & -0.012 & 0.005 & $2.62 \mathrm{E}-02$ & 0.58 & -0.012 & 0.003 & $1.44 \mathrm{E}-05$ \\
\hline 19 & 1457211 & cg14559388 & $A P C 2$ & Body & -0.003 & 0.001 & $3.49 E-05$ & -0.002 & 0.001 & $4.52 E-02$ & 0.05 & -0.003 & 0.001 & $4.98 E-06$ \\
\hline 19 & 1465207 & cg04624885 & $A P C 2$ & Body & -0.016 & 0.003 & $3.08 E-07$ & -0.003 & 0.003 & $4.43 E-01$ & 0.66 & -0.010 & 0.002 & $1.56 \mathrm{E}-05$ \\
\hline 19 & 1472936 & cg19870717 & $A P C 2$ & $3^{\prime}$-UTR & -0.010 & 0.002 & $1.81 E-08$ & -0.006 & 0.003 & $4.46 \mathrm{E}-02$ & 0.37 & -0.009 & 0.002 & $4.64 \mathrm{E}-09$ \\
\hline 19 & 1473042 & cg16613938 & APC2 & $3^{\prime}$-UTR & -0.017 & 0.003 & $1.15 E-07$ & -0.011 & 0.006 & $7.00 E-02$ & 0.71 & -0.016 & 0.003 & $3.05 E-08$ \\
\hline 19 & 1473179 & cg23291200 & $A P C 2$ & $3^{\prime}$-UTR & -0.011 & 0.002 & $7.81 E-08$ & -0.008 & 0.003 & $3.92 E-03$ & 0.63 & -0.010 & 0.002 & $1.72 E-09$ \\
\hline 19 & 51415450 & cg13793157 & $K L K 4$ & & -0.009 & 0.002 & $2.85 E-04$ & -0.009 & 0.005 & $5.41 \mathrm{E}-02$ & 0.53 & -0.009 & 0.002 & $4.00 E-05$ \\
\hline 19 & 51415452 & cg10078829 & $K L K 4$ & & -0.007 & 0.002 & $1.89 E-04$ & -0.007 & 0.003 & $3.57 \mathrm{E}-02$ & 0.40 & -0.007 & 0.002 & $1.84 \mathrm{E}-05$ \\
\hline
\end{tabular}

CHR, chromosome; Coef, regression coefficient from statistical model; gene, mapped or nearest gene (within $10 \mathrm{Mb}$ ) symbol using the UCSC database and Snipper software; gene group, gene region feature category (UCSC with verification); $P$, uncorrected $P$ value from statistical model; UTR, untranslated region; weighted mean beta, average of the mean methylation beta values for MoBa and feature category (UCSC with verification), $P$, uncorrected

Selection limited to genes with at least two CpGs at FDR significance that were prioritized for discussion.

Meta-analysis of results for 1,275 MoBa participants and 713 Generation R participants. Robust linear regression models adjusted for maternal age, maternal education, maternal sustained smoking during pregnancy, parity and batch. Results sorted by the chromosome and position of the CpG sites listed. For complete list of CpGs differentially methylated in relation to maternal folate and for results from meta-analysis models unadjusted for covariates and adjusted for covariates and cell type see Supplementary Data 1 (sorted by $P$ value) and 2 (sorted by chromosome, position). Supplementary Data include columns for mapped and nearest gene for each $\mathrm{CpG}$.

folate, it is an excellent marker of folate status. Homocysteine was strongly correlated with maternal plasma folate in $\mathrm{MoBa}$ (Spearman correlation $=-0.49, \quad P<0.001$ ) and moderately correlated in Generation $\mathrm{R}$ (Spearman correlation $=-0.24$, $P<0.001$ ), making it challenging to estimate independent effects. Given these various factors, inclusion of homocysteine in the model led to a moderate change in the coefficients for folate in relation to methylation (median change $10.7 \%, 25-75$ th percentile $5.8-17.2 \% . N=1,931$ subjects) and only 137 (31\%) CpGs remained statistically significant $\left(P<1.13 \times 10^{-4}\right.$, correction for 443 tests).

We also examined whether the associations with methylation seen for maternal folate levels are also seen for newborn folate levels in a subset of 572 subjects in Generation R. Thus, this analysis is not well powered compared with our maternal folate analysis with 1,988 subjects. However, of the 443 FDR-significant findings for maternal folate in the meta-analysis there were 60 (14\%) with nominal $P$ values $<0.05$ for newborn folate which is higher than the $5 \%$ expected by chance alone (Kolmogorov $\left.P<1.2 \times 10^{-13}\right)$. This supports the interpretation that some similar loci are differentially methylated in response to infant folate, although we were severely underpowered to address this properly.

Pathway analysis. Pathway analysis with the FDR-significant CpGs showed strong and consistent enrichment of fundamental development pathways and of neurodevelopmental pathways (Supplementary Tables 2-4). The biological processes implicated from the DAVID pathway analysis included cell development, embryonic morphogenesis, development, regulation of multicellular organismal processes, cell-cell signalling, embryonic development, forebrain development and, notably, neural tube development (Supplementary Table 2). Ingenuity Pathway Analysis (IPA) results indicated pathways related to nervous system development and function, cell-cell signalling and basic developmental processes (Supplementary Table 3). Gene ontology enrichment analysis and visualization tool results included pathways related to the synaptic signalling, cell-cell signalling, regulation of cAMP biosynthetic process, single-organism behaviour, single-organism signalling, signalling, regulation of gastrulation and the regulation of nervous system development (Supplementary Table 4).

Methylation expression analysis. Of the 365 CpGs associated with folate that we were able to match to a gene transcript $( \pm 250 \mathrm{~kb}), 43 \mathrm{CpGs}$ were significantly associated with altered expression of nearby genes $\left(P_{\mathrm{BH}}<0.1\right)$. For most CpGs, increased methylation was associated with decreased gene expression (Supplementary Table 5).

\section{Discussion}

Our study is the largest to date using the Illumina $450 \mathrm{~K}$ epigenome-wide platform to evaluate the impact of maternal plasma folate levels during pregnancy on DNA methylation in newborns. We meta-analysed results from two population-based birth cohort studies in Northern Europeans that measured DNA methylation using the same platform. We observed epigenomewide FDR-significant associations between maternal plasma folate and DNA methylation in cord blood at $443 \mathrm{CpGs}$. 
It is notable that many of the implicated genes have functional relevance to various developmental pathways. Some are relevant to NTDs, the indication for maternal folic acid supplementation, and others to distinct developmental conditions that have not been previously associated with maternal folate levels. Additional genes we identified have been implicated in conditions where there is some concern about possible adverse effects of higher folate levels, such as breast cancer progression ${ }^{16}$. Due to the large number of genes significantly differentially methylated in relation to folate (Supplementary Data 1 and 2), we focus this discussion primarily on genes with two or more $\mathrm{CpGs}$ at genome-wide significance after FDR correction $\left(P_{\mathrm{BH}}<0.05\right)$ where at least one $\mathrm{CpG}$ is within the gene (Table 2).

We observed the largest number (nine) of statistically significant $\mathrm{CpGs}$ mapping to the gene adenomatosis polyposis coli 2 gene (APC2). APC2 is expressed in both human fetal and adult brain ${ }^{17}$ and in the peripheral nervous system ${ }^{18}$. It plays a critical role in the brain development in several model systems ${ }^{19}$. APC2 may also play a role in cancer aetiology. A homologue of the tumour suppressor gene $A P C^{20}, A P C 2$, is involved in the regulation of the Wnt signalling pathway, which impacts both normal development and tumorigenesis ${ }^{21}$. Studies in mice have reported associations between periconceptional maternal folate and methylation of $A P C$ genes $^{22}$. In two human breast cancer lines, folate leads to methylation-mediated silencing of $A P C$ and other tumour suppressor genes, raising concern about the risk of tumour progression ${ }^{23}$. Thus, folate-related methylation of APC2 during fetal development could impact both pathways of neurodevelopment and carcinogenesis.

GRM8 encodes a glutamate receptor that interacts with L-glutamate, the major excitatory neurotransmitter in the central nervous system. Glutamatergic neurotransmission is ubiquitous in normal brain function ${ }^{24}$ and is perturbed in various neuropathologies. In humans, copy-number variations of GRM8 have been associated with neurodevelopmental disorders such as attention-deficit hyperactivity disorder ${ }^{25}$ and autism spectrum disorder ${ }^{26}$

A number of genes we identified as differentially methylated in newborns in relation to maternal folate are known to harbour mutations that have been causally implicated in various developmental abnormalities other than NTDs, the indication for folic acid supplementation in pregnancy. These include several with two or more statistically significant CpGs (Table 2) such as SLC16A12, implicated in juvenile cataracts with microcornea and renal glucosuria $^{27}$; and $K L K 4$, implicated in the dental malformation amelogenesis imperfecta ${ }^{28}$. Mutations in LHX1 have been associated with abnormalities in uterine development ${ }^{29}$, and recent evidence suggests an important role in retinal development ${ }^{30}$. Several genes with one $\mathrm{CpG}$ at genome-wide statistical significance (Supplementary Data 1 and 2) also harbour mutations that are causal for various development malformations. These include $I H H$ involved in skeletal malformations, $\mathrm{ROBO}$ involved in horizontal gaze palsy with progressive scoliosis, PCSK9 involved in familial hypercholesterolemia, FAM83H related to amelogenesis imperfecta type 3 and GJA3 associated with congenital cataracts. Taken together, these findings suggest a role for periconceptional folate levels in birth defects not previously known to be related to this nutrient.

Our agnostic evaluation of maternal folate levels and DNA methylation in newborns also identified genes related to various neurologic diseases. Genetic variation in OPCML and $P R P H$ has been associated with the neurodegenerative disease amyotrophic lateral sclerosis ${ }^{31,32}$. In genome-wide association studies, CSMD1 has been associated with schizophrenia and autism ${ }^{33}$.

Some previous studies of folate and methylation have examined the $H 19$ imprinted region ${ }^{11,34}$. We identified three significant CpGs located 45-48-Kb upstream of $H 19$ among 77 CpGs on the platform that are within 48 up- or downstream of H19.

The largest number of statistically significant associations at any locus, 31, are on chromosome 12 and, based on our extended annotation, are nearest to $A L G 10$. Two CpGs are 262-573-kb upstream; the other $29 \mathrm{CpGs}$ are 261-573-kb downstream. None are in $A L G 10$. Most are in a CpG island near the centromere and there are no features that suggest functional impact.

In the only previous study using the $450 \mathrm{~K}$ platform, Amarasekera et al ${ }^{12}$ reported differential methylation in relation to maternal folate in a 923-bp region on chromosome $6,3-\mathrm{kb}$ upstream of ZFP57. Our studies differ in sample sizes, design and analysis methods. However, when we evaluate the $20 \mathrm{CpG}$ s that map to ZFP57, we find 5 with uncorrected $P$ values of 0.05 or smaller-more than would be expected by chance alone. Thus, our data provide support for association at this locus.

From correlation analysis of $450 \mathrm{~K}$ methylation data and gene expression in white blood cells in adults, after correction for multiple testing, $43 \mathrm{CpGs}$ that we implicated in relation to maternal folate were also related to expression of nearby genes (Supplementary Table 5). Although correlation of $450 \mathrm{~K}$ methylation with gene expression in the same newborn samples would have been preferable, we were only able to examine correlations in a population of Dutch adults. The most statistically significant correlation between methylation and gene expression was observed for the gene PRSS21 (protease serine 21 (testisin)); four CpGs were both significantly associated with maternal folate (Table 2) and expression of this gene (Supplementary Table 5). PRSS21 is a tumour suppressor gene silenced by aberrant methylation in testicular germ cell tumours ${ }^{35}$. Testicular germ cell tumours are diagnosed in early adulthood and can manifest as early as 15 years of age. Prenatal origin of this tumour has been proposed $^{36}$; perhaps, methylation in utero, influenced by maternal folate levels, could play a role in this pathogenesis.

Because other important factors in one-carbon metabolism could potentially explain associations between folate levels and DNA methylation in cord blood, we performed various sensitivity analyses (Supplementary Data 3). On the basis of these analyses, vitamin $\mathrm{B} 12$ does not confound the folate-methylation association. This lack of confounding by B12 should extend to other B vitamins such as B6 and riboflavin that are present in multivitamins along with B12. We did not have data in both studies on choline, a nutrient that can serve as a source of one-carbon units. However, in $\mathrm{MoBa}$, where choline was measured, there was no correlation with folate levels (Spearman correlation $=-0.034, P=0.23$ ) and thus choline should not confound associations between folate and methylation. Vitamin $\mathrm{D}$ is not part of the one-carbon metabolism cycle but might impact methylation by other mechanisms ${ }^{37}$. We performed analyses in a subsample taking vitamin $\mathrm{D}$ into account as proxy for intake of other supplements or possibly healthy dietary patterns and observed no major differences in results. Adjustment of the folate-methylation association for homocysteine, a product formed in one-carbon metabolism that is itself an excellent marker of folate status, resulted in a substantial reduction in the number of statistically significant findings. Although caution is required, both because folate and homocysteine are correlated, and because they operate together in a cycle rather than a clear unidirectional pathway, this attenuation could be interpreted as homocysteine, at least in part, mediating some of the associations between folate and methylation.

Given the role of folate as a major provider of methyl groups in the one-carbon metabolism pathway, our finding of reduced methylation with higher folate at the majority of the implicated CpGs may seem counterintuitive. However, methyl groups from the one-carbon metabolism pathway are used in a range of 
biological processes and the complex interactions of these systems may not necessarily result in linear relationships. Indeed, there is evidence that effects of folate on folate-dependent enzymes may switch directions at the higher intracellular concentrations that may accompany folic acid supplementation ${ }^{38}$. Folic acid, in vitamin supplements or food fortification, is a synthetic folate with possible effects that differ from those of natural occurring folate species. There is recent evidence that folic acid interferes with the inhibitory effect of $S$-adenosylmethionine (SAM) on methylenetetrahydrofolate reductase (MTHFR) ${ }^{39}$ and may inhibit MTHFR activity, thereby reducing the amount of 5-methyltetrahydrofolate, SAM and the SAM/S-adenosylhomocysteine (SAH) ratio ${ }^{40}$. The SAM/SAH ratio has been referred to as the methylation potential; low SAM/SAH ratio may decrease DNA methylation. This may explain the inverse relationship we observe in our study but additional research is needed to more fully explain the complex biochemistry behind these observations. Of note, inverse correlations between prenatal folate status and DNA methylation at differentially methylated loci have been identified in the other population studies including Hoyo et $a .^{34}$ and Amarasekera et al. ${ }^{12}$

Although the health outcomes that have been related to folic acid supplementation involve target tissues such as the nervous system, we only had cord blood available for assessment of methylation. We do not know whether differential methylation at the sites that we observed in cord blood would be observed in relevant target tissues. While divergence in epigenetic patterns is critical for cell-type regulation, there is also evidence of similarities in patterns among some tissues ${ }^{41-43}$. We do not have data on methylation at older ages and thus the question of whether the differential methylation at these loci seen at birth in relation to maternal folate persists to later childhood would need to be addressed in future studies.

We measured folate using two different platforms in the two studies. Both are valid methods for the measurements of folate. Levels were reasonably similar although slightly higher in Generation R, which could reflect a difference in the platforms, differences in folate intake or the earlier timing of measurements in Generation R ( 12-week gestation in Generation R versus $\sim 18$-week gestation in MoBa). Nonetheless, the top findings were consistent in both cohorts and thus robust to differences in measurement platforms. This may increase their generalizability to other populations.

One-carbon metabolism is a complex pathway with influences from multiple genetic, hormonal and environmental factors. Despite our attempt to account for other important dietary intake involved in one-carbon metabolism, other supplementation and genetic variants, residual confounding could still be present and influence the observed associations of folate levels in pregnancy with methylation at birth.

The MoBa and Generation $\mathrm{R}$ cohorts offer a unique opportunity to study the epigenetic effect of folic acid supplementation in the absence of food supply fortification. It is possible that results may differ in populations exposed to fortification.

We identified multiple novel genes not previously implicated in biological responses to folate. Many of the implicated genes have functional relevance to various developmental pathways, including the nervous system. Some of these are relevant not only to NTDs, the indication for maternal folic acid supplementation, but also to other developmental abnormalities that have not been previously associated with maternal folate levels. The associations between periconceptional folate and these conditions are difficult to study because the abnormalities are rare and both supplementation and fortification are now widespread. Other genes identified are implicated in conditions where concern exists about possible adverse effects of higher folate levels, such as breast cancer progression ${ }^{16}$. These findings may provide new insights into mechanisms for the associations between maternal folate status and health outcomes in the offspring. Given that food fortification programs have greatly increased the folate status of the population, greater understanding of the biological effects of this nutrient is important. The large number of novel genes identified using our genome-wide methylation approach may shed light on the protean effects of folate on human health.

\section{Methods}

Study populations. This analysis included participants of the Norwegian Mother and Child Cohort Study (MoBa $)^{44,45}$ and participants of the Generation R Study from the Netherlands. The study populations and cohort-specific methods described below are more extensively detailed in the Supplementary Information (Supplementary Note). The MoBa participants were mother-offspring pairs from a substudy measuring maternal plasma folate during pregnancy ${ }^{46}$. The Generation $\mathrm{R}$ Study is a population-based prospective cohort study from fetal life onwards ${ }^{47,48}$. For this analysis, information on plasma folate and DNA methylation was available for 1,289 mothers and their children from the MoBa study (1,275 with complete covariate data) and 790 Caucasian mothers, and their children from the Generation R Study (713 with complete covariate data).

The MoBa study was approved by the Regional Committee for Ethics in Medical Research, the Norwegian Data Inspectorate and the Institutional Review Board of the National Institute of Environmental Health Sciences, USA, and written informed consent was provided by all mothers participating. The Generation R Study has been approved by the Medical Ethical Committee of the Erasmus MC, University Medical Center Rotterdam, Netherlands and written consent was obtained from participating parents of their children.

Maternal plasma folate measurements. Both cohorts measured maternal plasma folate during pregnancy. For $\mathrm{MoBa}$, maternal blood samples were drawn during pregnancy (median weeks gestation $=18$ weeks, $25-75$ th percentile $=16-21$ weeks) in EDTA-lined tubes, centrifuged within $30 \mathrm{~min}$ after collection and stored at $4{ }^{\circ} \mathrm{C}$ in the hospital where they were collected. Samples were then shipped overnight to the Biobank of MoBa at the Norwegian Institute of Public Health in Oslo. Upon receipt (1-2 days after blood collection), plasma was aliquoted onto polypropylene microtiter plates, sealed with heat-sealing foil sheets and stored at $-80^{\circ} \mathrm{C}$. Plasma folate concentration was measured at Bevital AS (www.bevital.no) by microbiological assay, using a chloramphenicol-resistant strain of Lactobacillus case $^{49}$, which measures biologically active folate species, predominantly 5-methyltetrahydrofolate. The coefficient of variation (CV) for this assay corresponds to $4 \%$ within day and $5 \%$ between days, at population median.

For the Generation R cohort, venous blood samples were drawn at enrolment of the mothers in early pregnancy (median weeks gestation $=12.9$ weeks; $25-75$ th percentile $=12.1-13.9$ weeks) and stored at room temperature for a maximum of $3 \mathrm{~h}$. Samples were transported to a laboratory facility of the regional laboratory in Rotterdam, Netherlands (Star-Medisch Diagnostisch Centrum) for additional processing and storage at $-80^{\circ} \mathrm{C}$. The samples were analysed at the Department of Clinical Chemistry at the Erasmus MC, University Medical Center Rotterdam, Netherlands. After thawing, folate concentrations were analysed using an immunoelectrochemoluminence assay on the Architect System (Abbott Diagnostics BV). Between-run CVs for plasma measurements were $8.9 \%$ at $5.6 \mathrm{nmoll}^{-1}, 2.5 \%$ at $16.6 \mathrm{nmoll}^{-1}$ and $1.5 \%$ at $33.6 \mathrm{nmoll}^{-1}$ with an analytic range of $1.8-45.3 \mathrm{nmoll}^{-1}$ for plasma folate.

Covariates. Each cohort had information on maternal age, education and parity from questionnaires completed by the mother or from birth registry records. Maternal smoking during pregnancy was ascertained with questionnaires (both cohorts) and cotinine levels (MoBa). Plasma levels of vitamin B12, vitamin D and total homocysteine from samples taken during pregnancy were available for both cohorts. Mothers in both cohorts were genotyped for two SNPs in the (NAD $(P) H)$ MTHFR gene, rs1801131 and rs1801133. Additional detail on these measurements is in the Supplementary Information (Supplementary Note).

DNA methylation measurements. DNA was extracted from cord blood and bisulfite conversion performed (EZ-96 DNA Methylation kit, Zymo Research Corporation, Irvine, USA). Samples were processed with Illumina's Infinium HumanMethylation450 BeadChip (Illumina Inc., San Diego, USA) followed by cohort-specific laboratory quality control. Each cohort calculated the methylation betas, and normalized the betas using a published method ${ }^{50,51}$.

Estimation of cell-type proportions. Both the MoBa and Generation R studies estimated cell-type proportion with the Houseman method ${ }^{52}$ as implemented in the $R$ minfi package ${ }^{53}$ using the Reinius et al. data set for reference ${ }^{54}$. Cell-type correction was applied by including the six estimated cell-type proportions as covariates in cohort-specific statistical models. 
Cohort-specific statistical analyses. The cohort-specific statistical models were run independently. For each cohort, we used robust linear regression models in $R^{55}$ to evaluate the association between natural $\log$-transformed maternal plasma folate and cord blood DNA methylation for each probe while accounting for potential heteroskedasticity and/or influential outliers. Models were adjusted for maternal age, education, smoking during pregnancy, parity and for batch effects (adjustment for plate in Generation R, correction using $\mathrm{ComBat}^{50}$ in $\mathrm{MoBa}$ ). Additional correction for study design was done in MoBa (whether the participant was in the MoBal or MoBa2 data set). Sex of the child was not expected to be associated with maternal plasma folate and was therefore not included as a covariate in the analyses. The adjustment variables were selected on a priori considerations and because they were also associated with maternal plasma folate levels at $P<0.05$.

Meta-analysis. The probe-specific quality control resulted in $473,731 \mathrm{CpGs}$ in the MoBa cohort and 436,013 CpGs in the Generation R cohort. The meta-analysis was limited to the 425,749 CpGs common to both cohorts. An additional 5,844 CpGs were excluded for having a SNP mapping to the last five nucleotides of the probe sequence and with a minor allele frequency $\geqslant 5 \%$ in the CEU (Utah residents with North and Western European ancestry) population, curated by $1000 \mathrm{G}$ projects (http://www.1000genomes.org/, 06/2011 release, 87 individuals), HapMap project (http://hapmap.ncbi.nlm.nih.gov/, release 28, 8/2010, 174 individuals) and dbSNP (http://www.ncbi.nlm.nih.gov/projects/SNP/, build 134, 8/2011, 116 individuals). This left 419,905 CpGs for the final meta-analyses.

Fixed-effect meta-analysis weighted by the inverse of the variance was completed using $M E T A L^{56}$. Multiple testing was accounted for by using the FDR procedure by Benjamini and Hochberg $(\mathrm{BH})^{57}$. For each $\mathrm{CpG}$, the resulting $\mathrm{BH}$ corrected $P$ values are denoted by $P_{\mathrm{BH}}$. CpGs with $P_{\mathrm{BH}}<0.05$ were considered statistically significant. CpGs that were statistically significant based on the more stringent Bonferroni correction (uncorrected $P<1.19 \times 10^{-7}$ to account for 419,905 tests) were noted. We present the covariate-adjusted model without cell-type adjustment as the primary results. In the Supplementary Information, we present results additionally adjusted for cell type and results without covariate adjustment.

\section{Sensitivity analyses. We performed sensitivity analyses to assess whether the} associations observed between folate and methylation might be explained by levels of vitamin B12, a dietary co-factor involved in regulating carbon unit bioavailability. Vitamin B12 is generally present in multivitamins that pregnant women in our studies may have taken in addition to, or in lieu of, separate folic acid supplements. Multivitamin supplements containing B12 typically contain other B vitamins including vitamin B6, which is also involved in one-carbon metabolism. Because mothers with higher folate levels may have higher intakes of other vitamin supplements not involved in the one-carbon metabolism pathway, or healthier diets in general, we also performed separate analyses adjusting for maternal plasma vitamin D levels during pregnancy. We also examined two SNPs in MTHFR involved in modulation of one-carbon metabolism: rs1801131 and rs1801133 (refs 14,15). We evaluated the impact of adjustment for total homocysteine on the association between maternal plasma folate and DNA methylation in newborns. Finally, we examined whether the associations with methylation seen for maternal folate levels are also seen for newborn folate levels in a subset of 572 subjects in Generation R.

Pathway analysis. To better understand the functional relationships between differentially methylated CpGs, we evaluated the FDR-significant CpGs with pathway analysis using three independent software programs. First, gene ontology analysis was performed using the IPA (www.ingenuity.com) based on the content version of 21249400 (release date: 22 September 2014). For a given category in IPA, Fisher's exact test was used to measure the probability that the category was randomly associated $(P<0.05$ defined as significantly enriched). Second, the NIAID's DAVID Bioinformatics Resources 6.7 (ref. 58) was used to analyse enrichments in main categories: biological process, cellular component, molecular function and KEGG pathway. Third, we used gene ontology enrichment analysis and visualization tool ${ }^{59}$ to identify the most informative terms that are significantly enriched.

\section{Methylation expression analysis. We evaluated the association between} methylation and quantitative levels of gene expression for our top CpGs. We used messenger RNA gene expression and $450 \mathrm{~K}$ methylation data both from white blood cells from adults over 45 years of age in the Rotterdam Study, a populationbased prospective cohort study in Rotterdam, the Netherlands. Among the 443 FDR-significant CpGs associated with folate, we were able to match $365 \mathrm{CpGs}$ to a gene transcript in our gene expression data set within a region of 250-kb upstream or downstream of the CpG (total region $500 \mathrm{~kb}$ ). We analysed the associations of these CpGs with expression levels of the corresponding gene transcripts.

\section{References}

1. Miller, J. W. \& Ulrich, C. M. Folic acid and cancer--where are we today? Lancet 381, 974-976 (2013).

2. De-Regil, L. M., Fernández-Gaxiola, A. C., Dowswell, T. \& Peña-Rosas, J. P. Effects and safety of periconceptional folate supplementation for preventing birth defects. Cochrane Database Syst. Rev. CD007950 (2010); doi:10.1002/ 14651858.CD007950.pub2.

3. Bortolus, R. et al. Prevention of congenital malformations and other adverse pregnancy outcomes with $4.0 \mathrm{mg}$ of folic acid: community-based randomized clinical trial in Italy and the Netherlands. BMC Pregnancy Childbirth. 14, 166 (2014).

4. Barua, S., Kuizon, S. \& Junaid, M. A. Folic acid supplementation in pregnancy and implications in health and disease. J. Biomed. Sci. 21, 77 (2014).

5. O'Neill, R. J., Vrana, P. B. \& Rosenfeld, C. S. Maternal methyl supplemented diets and effects on offspring health. Front. Genet. 5, 289 (2014).

6. Choumenkovitch, S. F. et al. Folic acid intake from fortification in United States exceeds predictions. J. Nutr. 132, 2792-2798 (2002).

7. Nakouzi, G. A. \& Nadeau, J. H. Does dietary folic acid supplementation in mouse NTD models affect neural tube development or gamete preference at fertilization? BMC Genet. 15, 91 (2014).

8. Fox, J. T. \& Stover, P. J. Folate-mediated one-carbon metabolism. Vitam. Horm 79, 1-44 (2008).

9. Begin, P. \& Nadeau, K. C. Epigenetic regulation of asthma and allergic disease Allergy Asthma Clin. Immunol. 10, 27 (2014).

10. Chang, H. et al. Tissue-specific distribution of aberrant DNA methylation associated with maternal low-folate status in human neural tube defects. J. Nutr. Biochem. 22, 1172-1177 (2011).

11. Loke, Y. J. et al. Association of maternal and nutrient supply line factors with DNA methylation at the imprinted IGF2/H19 locus in multiple tissues of newborn twins. Epigenetics 8, 1069-1079 (2013).

12. Amarasekera, M. et al. Genome-wide DNA methylation profiling identifies a folate-sensitive region of differential methylation upstream of ZFP57imprinting regulator in humans. FASEB J. 28, 4068-4076 (2014).

13. Devlin, B. \& Roeder, K. Genomic control for association studies. Biometrics $\mathbf{5 5}$, 997-1004 (1999).

14. Tsang, B. L. et al. Assessing the association between the methylenetetrahydrofolate reductase (MTHFR) $677 \mathrm{C}>\mathrm{T}$ polymorphism and blood folate concentrations: a systematic review and meta-analysis of trials and observational studies. Am. J. Clin. Nutr. 101, 1286-1294 (2015).

15. van der Valk, R. J. et al. Neonatal folate, homocysteine, vitamin B12 levels and methylenetetrahydrofolate reductase variants in childhood asthma and eczema. Allergy 68, 788-795 (2013).

16. Slattery, M. L. et al. MAPK genes interact with diet and lifestyle factors to alter risk of breast cancer: the breast cancer health disparities study. Nutr. Cancer 67, 292-304 (2015).

17. Roadmap Epigenomics, C. et al. Integrative analysis of 111 reference human epigenomes. Nature 518, 317-330 (2015).

18. Jarrett, C. R. et al. Human APC2 localization and allelic imbalance. Cancer Res. 61, 7978-7984 (2001).

19. Shintani, T. et al. APC2 plays an essential role in axonal projections through the regulation of microtubule stability. J. Neurosci. 29, 11628-11640 (2009).

20. Cancer Genome Atlas, N. Comprehensive molecular characterization of human colon and rectal cancer. Nature 487, 330-337 (2012).

21. van Es, J. H. et al. Identification of APC2, a homologue of the adenomatous polyposis coli tumour suppressor. Curr. Biol. 9, 105-108 (1999).

22. Sie, K. K. et al. Effect of maternal and postweaning folic acid supplementation on global and gene-specific DNA methylation in the liver of the rat offspring. Mol. Nutr. Food Res. 57, 677-685 (2013).

23. Lubecka-Pietruszewska, K., Kaufman-Szymczyk, A., Stefanska, B. \& Fabianowska-Majewska, K. Folic acid enforces DNA methylation-mediated transcriptional silencing of PTEN, APC and RARbeta2 tumour suppressor genes in breast cancer. Biochem. Biophys. Res. Commun. 430, 623-628 (2013).

24. Mukherjee, S. \& Manahan-Vaughan, D. Role of metabotropic glutamate receptors in persistent forms of hippocampal plasticity and learning. Neuropharmacology 66, 65-81 (2013).

25. Elia, J. et al. Genome-wide copy number variation study associates metabotropic glutamate receptor gene networks with attention deficit hyperactivity disorder. Nat. Genet. 44, 78-84 (2012).

26. Prasad, A. et al. A discovery resource of rare copy number variations in individuals with autism spectrum disorder. G3 (Bethesda) 2, 1665-1685 (2012).

27. Kloeckener-Gruissem, B. et al. Mutation of solute carrier SLC16A12 associates with a syndrome combining juvenile cataract with microcornea and renal glucosuria. Am. J. Hum. Genet. 82, 772-779 (2008).

28. Wang, S. K. et al. Novel KLK4 and MMP20 mutations discovered by wholeexome sequencing. J. Dent. Res. 92, 266-271 (2013).

29. Sandbacka, M. et al. TBX6, LHX1 and copy number variations in the complex genetics of Mullerian aplasia. Orphanet J. Rare. Dis. 8, 125 (2013).

30. Bedont, J. L. et al. Lhx1 controls terminal differentiation and circadian function of the suprachiasmatic nucleus. Cell Rep. 7, 609-622 (2014).

31. Corrado, L. et al. A novel peripherin gene (PRPH) mutation identified in one sporadic amyotrophic lateral sclerosis patient. Neurobiol. Aging 32, 552 e1-552 e6 (2011). 
32. Xie, T. et al. Genome-wide association study combining pathway analysis for typical sporadic amyotrophic lateral sclerosis in Chinese Han populations. Neurobiol. Aging 35, 1778 e9-1778 e23 (2014).

33. Hindorff, L. A. et al. A Catalog of Published Genome-Wide Association Studies. Available at http://www.genome.gov/gwastudies (accessed 15 May 2015).

34. Hoyo, C. et al. Methylation variation at IGF2 differentially methylated regions and maternal folic acid use before and during pregnancy. Epigenetics $\mathbf{6}$, 928-936 (2011)

35. Kempkensteffen, C. et al. Epigenetic silencing of the putative tumor suppressor gene testisin in testicular germ cell tumors. J. Cancer Res. Clin. Oncol. 132, 765-770 (2006).

36. Kristensen, D. G., Skakkebaek, N. E., Rajpert-De Meyts, E. \& Almstrup, K. Epigenetic features of testicular germ cell tumours in relation to epigenetic characteristics of foetal germ cells. Int. J. Dev. Biol. 57, 309-317 (2013).

37. Fetahu, I. S., Hobaus, J. \& Kallay, E. Vitamin D and the epigenome. Front. Physiol. 5, 164 (2014).

38. Smith, A. D., Kim, Y. I. \& Refsum, H. Is folic acid good for everyone? Am. J. Clin. Nutr. 87, 517-533 (2008).

39. Smith, D. E., Hornstra, J. M., Kok, R. M., Blom, H. J. \& Smulders, Y. M. Folic acid supplementation does not reduce intracellular homocysteine, and may disturb intracellular one-carbon metabolism. Clin. Chem. Lab. Med. 51, 1643-1650 (2013).

40. Christensen, K. E. et al. High folic acid consumption leads to pseudo-MTHFR deficiency, altered lipid metabolism, and liver injury in mice. Am. J. Clin. Nutr. 101, 646-658 (2015).

41. Ally, M. S., Al-Ghnaniem, R. \& Pufulete, M. The relationship between genespecific DNA methylation in leukocytes and normal colorectal mucosa in subjects with and without colorectal tumors. Cancer Epidemiol. Biomarkers Prev. 18, 922-928 (2009).

42. Byun, H. M. et al. Epigenetic profiling of somatic tissues from human autopsy specimens identifies tissue- and individual-specific DNA methylation patterns. Hum. Mol. Genet. 18, 4808-4817 (2009).

43. Talens, R. P. et al. Variation, patterns, and temporal stability of DNA methylation: considerations for epigenetic epidemiology. FASEB J. 24, 3135-3144 (2010).

44. Magnus, P. et al. Cohort profile: the Norwegian Mother and Child Cohort Study (MoBa). Int. J. Epidemiol. 35, 1146-1150 (2006).

45. Ronningen, K. S. et al. The biobank of the Norwegian Mother and Child Cohort Study: a resource for the next 100 years. Eur. J. Epidemiol. 21, 619-625 (2006).

46. Haberg, S. E. et al. Maternal folate levels in pregnancy and asthma in children at age 3 years. J. Allergy Clin. Immunol. 127, 262-264, 264 el (2011).

47. Jaddoe, V. W. et al. The Generation R Study: design and cohort update 2012. Eur. J. Epidemiol. 27, 739-756 (2012).

48. Kruithof, C. J. et al. The Generation R Study: Biobank update 2015. Eur. J. Epidemiol. 29, 911-927 (2014).

49. O'Broin, S. \& Kelleher, B. Microbiological assay on microtitre plates of folate in serum and red cells. J. Clin. Pathol. 45, 344-347 (1992).

50. Johnson, W. E., Li, C. \& Rabinovic, A. Adjusting batch effects in microarray expression data using empirical Bayes methods. Biostatistics 8, 118-127 (2007).

51. Teschendorff, A. E. et al. A beta-mixture quantile normalization method for correcting probe design bias in Illumina Infinium $450 \mathrm{k}$ DNA methylation data. Bioinformatics 29, 189-196 (2013).

52. Houseman, E. A. et al. DNA methylation arrays as surrogate measures of cell mixture distribution. BMC Bioinformatics 13, 86 (2012).

53. Aryee, M. J. et al. Minfi: a flexible and comprehensive Bioconductor package for the analysis of Infinium DNA methylation microarrays. Bioinformatics 30, 1363-1369 (2014).

54. Reinius, L. E. et al. Differential DNA methylation in purified human blood cells: implications for cell lineage and studies on disease susceptibility. PLoS ONE 7, e41361 (2012)

55. R Development Core Team (2010); R: A language and environment for statistical computing. R Foundation for Statistical Computing. Vienna, Austria. 3-900051-07-0 http://www.R-project.org/.

56. Willer, C. J., Li, Y. \& Abecasis, G. R. METAL: fast and efficient meta-analysis of genomewide association scans. Bioinformatics 26, 2190-2191 (2010).

57. Benjamini, Y. \& Hochberg, Y. Controlling for false discovery rate: a practical and powerful approach to multiple testing. J. R. Stat. Soc.B 57, 289-300 (1995).

58. Huang, da, W., Sherman, B. T. \& Lempicki, R. A. Bioinformatics enrichment tools: paths toward the comprehensive functional analysis of large gene lists. Nucleic Acids Res. 37, 1-13 (2009).

59. Eden, E., Navon, R., Steinfeld, I., Lipson, D. \& Yakhini, Z. GOrilla: a tool for discovery and visualization of enriched GO terms in ranked gene lists. $B M C$ Bioinformatics 10, 48 (2009).

\section{Acknowledgements}

This research was supported in part by the Intramural Research Program of the NIH, National Institute of Environmental Health Sciences (Z01-ES-49019). Additional funding support was provided by the NIH Office of Dietary Supplements. We acknowledge Shuangshuang Dai of Integrative Bioinformatics at the NIEHS and Jianping Jin of Westat for their expert data management and programming assistance. The Norwegian Mother and Child Cohort Study is supported by the Norwegian Ministry of Health and the Ministry of Education and Research, NIH/NIEHS (contract no. N01-ES-75558), NIH/NINDS (grant no.1 UO1 NS 047537-01) and the Norwegian Research Council/FUGE (grant no. 151918/ $\mathrm{S} 10$ ). We are grateful to all the participating families in Norway who take part in this ongoing cohort study. E.B. was supported by the Adam J Berry Memorial Scholarship administered by the Australian Academy of Science and the Foundation for the National Institutes of Health. The Generation R Study is conducted by the Erasmus Medical Center in close collaboration with the School of Law and Faculty of Social Sciences of the Erasmus University Rotterdam, the Municipal Health Service Rotterdam area, Rotterdam, the Rotterdam Homecare Foundation, Rotterdam and the Stichting Trombosedienst \& Artsenlaboratorium Rijnmond, Rotterdam. We gratefully acknowledge the contribution of children and parents, general practitioners, hospitals, midwives and pharmacies in Rotterdam. The study protocol was approved by the Medical Ethical Committee of the Erasmus Medical Centre, Rotterdam. We thank Mr Michael Verbiest, Ms Mila Jhamai, Ms Sarah Higgins, Mr Marijn Verkerk and Dr Lisette Stolk for their help in creating the EWAS database. The Generation R Study is made possible by financial support from the Erasmus Medical Center, Rotterdam, the Erasmus University Rotterdam and the Netherlands Organization for Health Research and Development. J.F.F. has received funding from the European Union's Horizon 2020 research and innovation programme under grant agreement No 633595. O.F. works in ErasmusAGE, a centre for aging research across the life course funded by Nestle Nutrition (Nestec Ltd), Metagenics Inc and AXA. Nestlé Nutrition (Nestec Ltd.), Metagenics Inc. and AXA had no role in design and conduct of the study, in the collection, management, analysis and interpretation of the data, or in the preparation, review and approval of the manuscript. A.D. received an additional grant from the Netherlands Organization for Health Research and Development (VENI 916.12.154) and the EUR Fellowship. V.W.V.J. received an additional grant from the Netherlands Organization for Health Research and Development (VIDI 016.136.361) and a Consolidator Grant from the European Research Council (ERC-2014-CoG-64916). L.D. received an additional grant from the Lung Foundation Netherlands (no 3.2.12.089; 2012). The generation and management of the Illumina $450 \mathrm{~K}$ methylation array data (EWAS data) for the Generation $\mathrm{R}$ Study was executed by the Human Genotyping Facility of the Genetic Laboratory of the Department of Internal Medicine, Erasmus Medical Center, the Netherlands. The EWAS data was funded by a grant to V.W.V.J. from the Netherlands Genomics Initiative (NGI)/ Netherlands Organisation for Scientific Research (NWO) Netherlands Consortium for Healthy Aging (NCHA; project nr. 050-060-810), and by funds from the Genetic Laboratory of the Department of Internal Medicine, Erasmus Medical Center.

\section{Author contributions}

All authors have seen and approved this manuscript. B.R.J. and H.T.d.D. were the primary authors and data analysts, and contributing equally under joint supervision with equal contribution from L.D. and S.J.L. All remaining authors helped interpret results and write the report.

\section{Additional information}

Accession codes: The complete genome wide meta-analysis results file has been deposited in the database of genotypes and phenotypes $(\mathrm{dbGaP})$ under the accession number phs001059.v1.p1. Access to individual-level Illumina HumanMethyl450 Beadchip data for the MoBa study dataset is available by application to the Norwegian Institute of Public Health using a form available on the English language portion of their website at http:// www.fhi.no/eway/. Specific questions regarding MoBa data access can be directed to Wenche Nystad: Wenche.Nystad@fhi.no. Requests for access to the individual level data for the Generation R study can be directed to Liesbeth Duijts: 1.duijts@erasmusmc.nl. For both studies the study management teams will verify with their local ethical committees that the applications are consistent with the consent provided. Applicants will need to obtain IRB approval or exemption from their local institutional review boards.

Supplementary Information accompanies this paper at http://www.nature.com/ naturecommunications

Competing financial interests: The authors declare no competing financial interests.

Reprints and permission information is available online at http://npg.nature.com/ reprintsandpermissions/

How to cite this article: Joubert, B. R. et al. Maternal plasma folate impacts differential DNA methylation in an epigenome-wide meta-analysis of newborns. Nat. Commun. 7:10577 doi: 10.1038/ncomms10577 (2016)

This work is licensed under a Creative Commons Attribution 4.0 International License. The images or other third party material in this article are included in the article's Creative Commons license, unless indicated otherwise in the credit line; if the material is not included under the Creative Commons license, users will need to obtain permission from the license holder to reproduce the material. To view a copy of this license, visit http://creativecommons.org/licenses/by/4.0/ 